\title{
An Energy and Spectral Efficient In Underwater Communication Using Magneto Inductive Channel
}

\author{
Kalpana.K, \\ II M.E ,PSN College of Engineering \& Technology, \\ Guide Mr.T.Rajesh, Assistant Professor, PSN College of Engineering \& Technology
}

\begin{abstract}
The analysis of Magneto Inductive communication technique due to underwater communication having multipath fading, dynamic channel and high propagation delay. The performance studies of 3D network covering 100 of meter of sea depth and few km square area show fully connected multi coil network with communication bandwidth extending from few to tens of $\mathrm{kHz}$. The low speed of sound in water, different attenuation characteristics and time varying multi-path fading make use of regular communication methods impractical. To get large area coverage and more bandwidth from sea area. In this work, highly power efficient and fully connected fuzzy scheduling is presented. It allows underwater channel more fair and efficient communication over larger distances.
\end{abstract}

Keywords: Underwater magnetic wireless communications, Magnetic-induction, induction coil, multiple access techniques, $3 D$ grid network

\section{INTRODUCTION}

The underwater communication is traditionally achieved by using acoustic radio frequency (RF) and optical channels. The major drawbacks of these channels prevent them from being fully reliable and practical technologies for underwater communication. In underwater RF communication, electromagnetic waves propagate over very short distances due to high levels of attenuation increasing with conductivity and frequency. Large antenna size, low operation frequencies and high transmission power are necessary. Similar problems are also observed in underground channels which are similar to underwater channel conditions, e.g., salty water content and dynamically changing conditions. In underwater optical channels, the source and destination nodes should form a directional link in a close proximity with high precision in pointing the narrow laser beams. Furthermore, the multiple scattering of light results in dispersion and creates the intersymbol interference. In underwater acoustic channels, which is the most promising technology used currently due to longer propagation distances underwater there are several major difficulties such as frequency-dependent propagation loss, multipath fading, high propagation delay, limited bandwidth, temporary losses of connectivity, extreme Doppler and wideband effects . It shows another very promising low-cost, robust and efficient method is the magnetoinductive (MI) wireless communication. Unlike acoustic channel, MI channel does not have high latency and it mitigates the challenges of dynamical conditions and high power consumptions by using simple, low cost and low power coils. The channel conditions depend on the permeability of the communication medium and a uniform channel is created in air, seawater and most types of soil and rock due to almost the same permeability. The feasible communication distance dramatically increases a waveguide. MI waveguides are introduced in based on coupled and capacitive loaded loops with analysis by using a circuit model and considering both nearest and distant neighbour effects with potential applications like power dividers, bends and couplers for 1D, 2D and 3D . Similar applications like guiding RF energy and general N-port devices including power splitters and directional couplers are presented in assuming lossless propagation and nearest neighbour coupling. Non-nearest neighbour interactions in MI waveguides are investigated. However, in none of these works, 3D MI wireless networks are considered with a communication theoretical modelling, performance analysis and application in Underwater communication networks.

MI communication is recently introduced in wireless underground communication (WUC) networks. Underground MI channel is theoretically analyzed and compared with RF channels and MI waveguides are utilized. In MI WUC in a district heating system is explored by analyzing a two-coil system in terms of antenna design. In deployment of waveguides to connect sensors is analyzed by optimizing the number of coils and increasing the network robustness.

MI waveguides are explored in terms of system architecture and operational framework to be used for underground pipeline monitoring. On the contrary, in this work, 3D MI wireless network topology is theoretically analyzed in terms of communication theoretical performance metrics by considering non-nearest neighbour interactions and realistic 3D grid underwater wireless networks are explored and numerically analyzed. There is a limited number of work using MI communications in underwater. The basic of wireless 
underwater communication may still seem far-fetched. Research has been active for over a decade on designing the method for wireless information transmitter underwater.

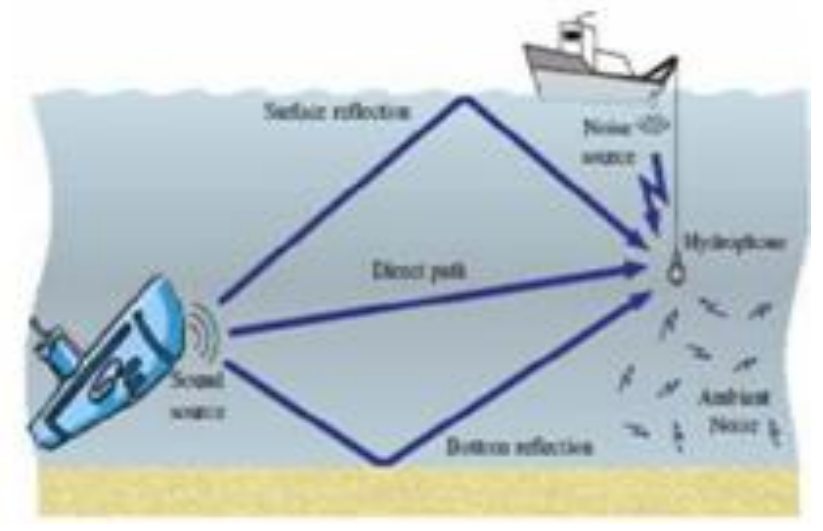

Fig 1: Example of multipath fading in underwater communication

To collect the information from remote undersea location. This information of high speed communication, link exist between remote end and surface .Underwater communication is a technique of sending and receiving message below water. There are several ways of employing such communication but the most common is using hydrophones. Under water communication is difficult due to factors like multi-path propagation, time variations of the channel, small available bandwidth and strong signal attenuation, especially over long ranges. In underwater communication there are low data rates compared to terrestrial communication, since underwater communication uses acoustic waves instead of electromagnetic waves.

Our Current underwater transmission techniques is primarily utilize sound waves for large distance at lower frequencies and the velocity of sound in water is approximately $1500 \mathrm{~m} / \mathrm{s}$ the resultant communications have problems with multi-path propagation and low bandwidth problems. The effect of multipath propagation and different signal propagation time at different rays and large inter symbol interference (ISI). Effective underwater acoustic communication system should reduce influence of the multipath propagation. A sound wave propagating underwater consists of alternating compressions and rarefactions of the water. These compressions and rarefactions are detected by a receiver, such as the human ear or a hydrophone, as changes in pressure. These waves may be man-made or naturally generated. A sound wave propagating underwater consists of alternating compressions and rarefactions of the water. These compressions and rarefactions are detected by a receiver, such as the human ear or a hydrophone, as changes in pressure. These waves may be man-made or naturally generated. The large impedance contrast between air and water (the ratio is about 3600) and the scale of surface roughness means that the sea surface behaves as an almost perfect reflector of sound at frequencies below $1 \mathrm{kHz}$. Sound speed in water exceeds that in air by a factor of 4.4 and the density ratio is about 820 .

\section{UNDERWATER MAGNETIC INDUCTION CHANNEL COMMUNICATION}

In this section, firstly, MI underwater channel is discussed. Next, the basic two-coil channel in a 3D environment is modelled by using the general mutual inductance calculations and equivalent circuit methods. Then, for a general multi coil 3D topology, the power loss performance is theoretically modelled by fully exploiting the inter-coil mutual inductance relationships.

\section{A. Underwater magneto inductive channel networking:}

Induction coil sensors are one of the oldest types of magnetic sensors Fine-to-coarse underwater in down sampling - Underwater physical medium is a challenging environment for using RF signal. It is due to some attenuation, power, delay, multi path fading.

- Using Magneto-inductive technique is stands strong alternative paradigm in independence of environmental impairment including fading, dynamic channel propagation delay in acoustic waves .

- MI techniques having low cost, easily deployable and flexible antenna structures, high power efficient.

- Underwater communication networks (UWSN) composed transceiver and relay induction coils are presented.

- UWCNs are analyzed in terms of basic communication metrics. i.e., SNR, BER, connectivity and bandwidth. 


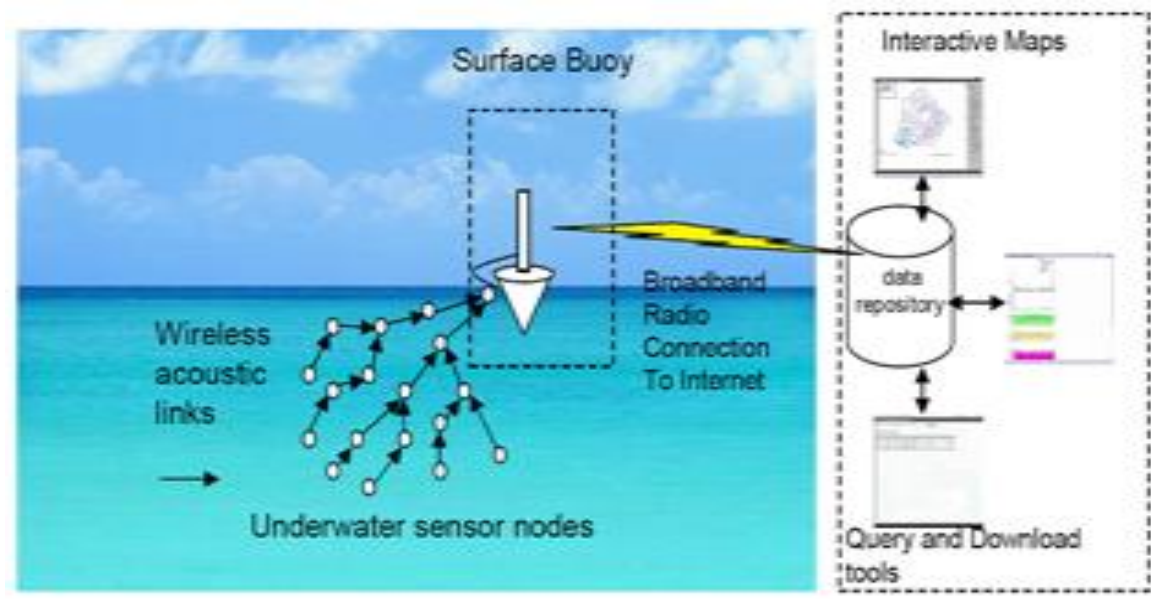

Fig 2: Network architecture of underwater communication

The above figure(2) shows that network architecture of magnetic induction. This Magnetic Induction Communication technology enables users of two-way radios to experience wireless mobility, wireless safety and wireless security with portable radio accessories. A remote control is a component of an electronics device, most commonly a television set, DVD player and home theatre systems originally used for operating the device wirelessly from a short line-of-sight distance. Remote control has continually evolved and advanced over recent years to include Bluetooth connectivity, motion sensor enabled capabilities and voice control.

The performance studies of 3D network covering 100 of meter of sea depth and few $\mathrm{km}$ square area show fully connected multi coil network with communication bandwidth extending from few to tens of $\mathrm{kHz}$.The wireless sensor networks satisfy these requirements desirable functions for sensor node includes ease of installation, self identification, self diagnosis, reliability, time awareness for coordination with other nodes .

The base band signals in a signals that can include frequency that are very near zero by comparison with its highest frequencies, for example a sound waveform can be considered as a base band signals where as a radio signals or any other modulated signals is not. The base band signals is low frequency signal which when modulated is transmitted on various channels.

\section{B. Underwater full duplex mutual inductive analysis:}

Underwater coils are open to disturbances resulting from water currents and wave effects making it necessary to analyze the mutual inductance relations among coils by considering the problem in 3D. The mutual inductance between two circular coils having 3D positional freedom with both lateral and angular misalignments.

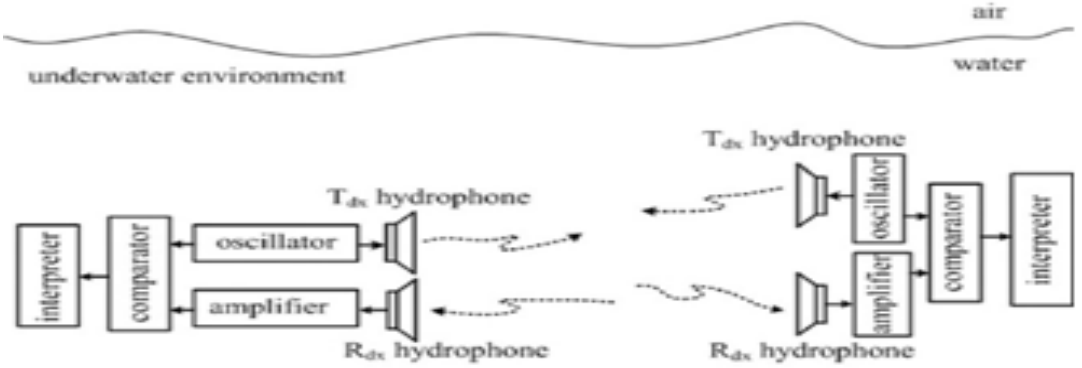

Fig 3: Full duplex active sensor communication system

$$
\begin{aligned}
& \mathrm{M}=(\mathrm{n} 2 \mathrm{t} 2 \mu 0 \sqrt{\mathrm{r}} 1 \mathrm{r} 2 / \pi) \times(\cos (\theta)-\mathrm{d} \cos (\varphi) / \mathrm{r} 2 \Psi(\mathrm{k}) / \mathrm{k} \zeta 3 \mathrm{~d} \varphi \\
& \text { where } \Psi(\mathrm{k})=(1-\mathrm{k} 2 / 2) \mathrm{K}(\mathrm{k})-\mathrm{E}(\mathrm{k}) \text {, } \\
& \zeta=1+\mathrm{d} 2 / \mathrm{r} 22-\eta, \mathrm{k} 2=4 \chi \zeta /((1+\chi \zeta) 2+\delta 2),
\end{aligned}
$$

Then, performance dependence on coil radius, wire diameter, capacitance and inter-coil distance is analyzed for grids of variable sizes and volumes. 'The coils weighting a few $\mathrm{kgs}$ can be hold in their positions by floating buoys for a dynamic network. In connectivity analysis, the term k-connectivity denotes a Network that each node connects to $\mathrm{k}$ nodes within the specific BER threshold. A fully connected network means that all 
the nodes can connect to each other within the BER threshold. Next, the realistic network topologies are used to analyze the SNR, BER, connectivity and bandwidth performance.

\section{PERFORMANCE ANALYSIS OF MI CHANNEL}

In this section, numerical and theoretical performance analyses of 3D grid underwater MI wireless networks are achieved. SNR, BER, connectivity and bandwidth performances are simulated for the networks extending along a line, e.g., sea shore, in shallow water and having rectangular area coverage in deep water.

\section{A. SNR, BER, Connectivity And Bandwidth Performance}

Two types of coverage topology are analyzed. Firstly, a rectangular prism with long range in $\mathrm{x}$ direction. e.g., along the shore of the sea, in shallow water is analyzed. The performance is analyzed in terms of inter-layer Distance, i.e., Lx, for the effect of the number of layers by Numerical simulation and comparison with single coil scheme Composed of one transmitter and receiver without any relays.
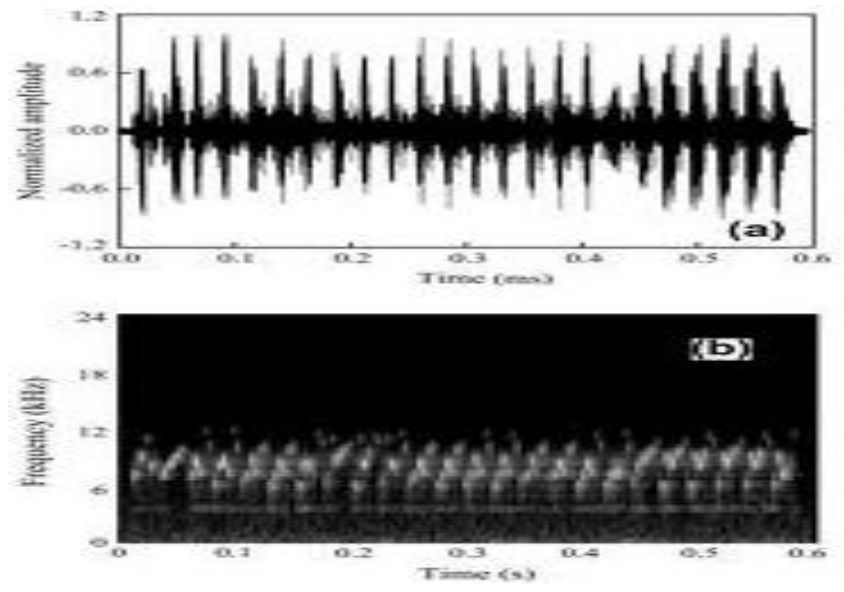

Fig 4: Decoder of output waveform in MI channel

Various transmitter and noise power levels are simulated. SNR performance of $\mathrm{Nx}=12$ layers grid network and its comparison with the single coil case are shown for nodes with varying interlayer distance ( $\mathrm{x}$ axis) in Fig.5 (a),(b)Maximum and minimum SNRs among the nodes having the specified $\mathrm{x}$ axis distance are compared. Single coil case simulation is performed by using only two coils at the respective positions of the grid network by removing all other coils except the transmitter significantly deteriorating performance dropping to $10 \mathrm{~dB}$ for the most distant position.

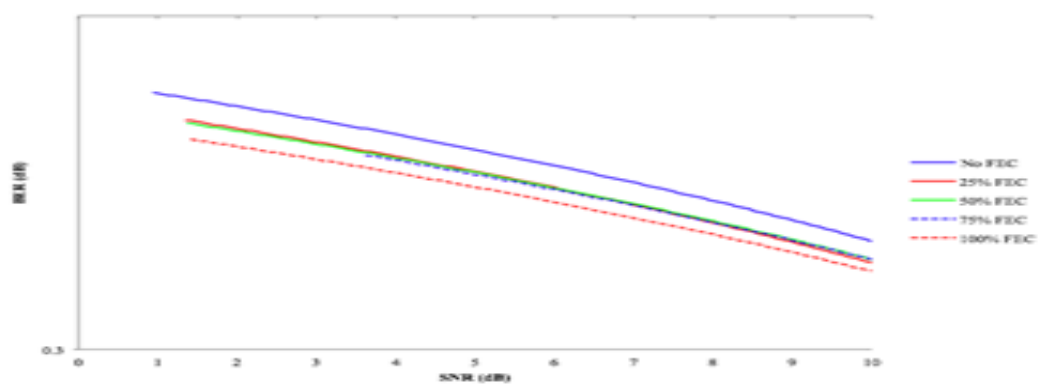

Fig 5: Performances of $\mathrm{Nx}=12$ layer grid network and single coil scheme

(no relay) for varying inter-layer distance (x axis) among the transmitter and the receiver nodes for (a) SNR Therefore similar to waveguides, grid network not only prevents SNR decay but also creates a connected network occupying a large 3D volume. 


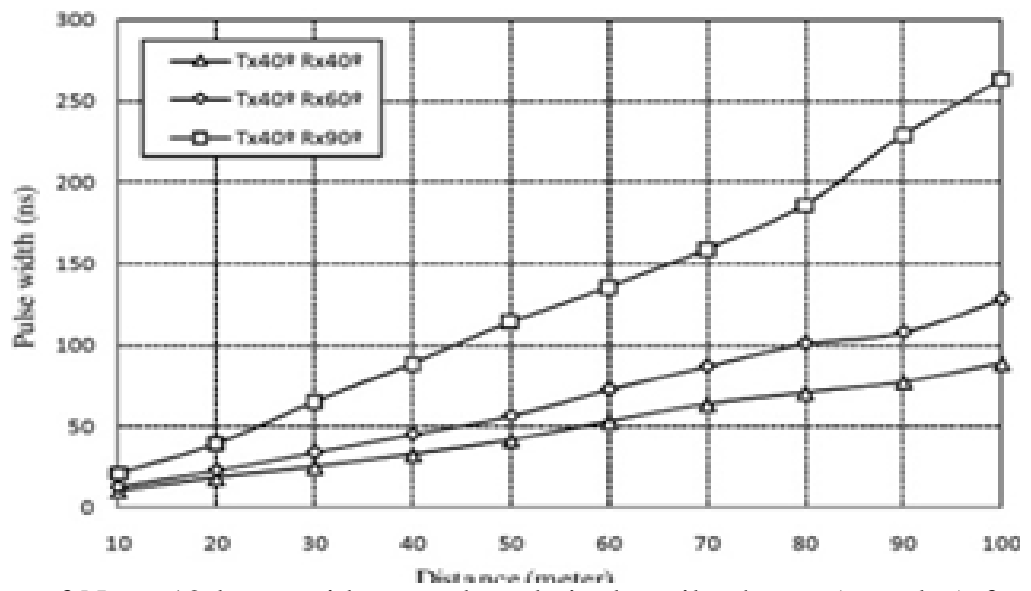

Fig 6: Performances of $\mathrm{Nx}=12$ layer grid network and single coil scheme (no relay) for varying inter-layer distance ( $\mathrm{x}$ axis) among the transmitter and the receiver nodes for $(\mathrm{b})$ bandwidth

elf inductance, i.e., L, and resistance, i.e., R, of a circular coil. Where R0 is resistance per meter depending on the type of the wire and its diameter. In Fig. 5(b), $3 \mathrm{~dB}$ bandwidth of the links between nodes having varying Lx is shown. It is observed that MI grid network has links with distance dependent bandwidth decreasing and saturating with the distance. Single coil case and grid network have around $\approx 60$ and $30 \mathrm{KHz}$ bandwidths among the nodes with layer distance $\mathrm{Lx} \approx 36 \mathrm{~m}$, respectively. As the distance increases, single coil case allows $\approx 4 \mathrm{KHz}$ communication while the grid network allows a much better $\approx 10 \mathrm{KHz}$ bandwidth showing the advantage of forming MI networks.

The above figure (7) shows that pilot insertion of plot 1,2,3 in MI channel, BER of the worst performance link for varying Pt and physical size of the network .

\section{B. Performance Dependence on Coil Parameters \& Grid Size}

A detailed theoretical analysis of the dependenceof the power loss on coil radius, wire diameter, capacitance and the grid inter-coil distance is presented. Furthermore, an approximation is given for the power loss between transmitter and receiver by clarifying the proportionality of the power loss on coil radius, wire diameter, capacitance
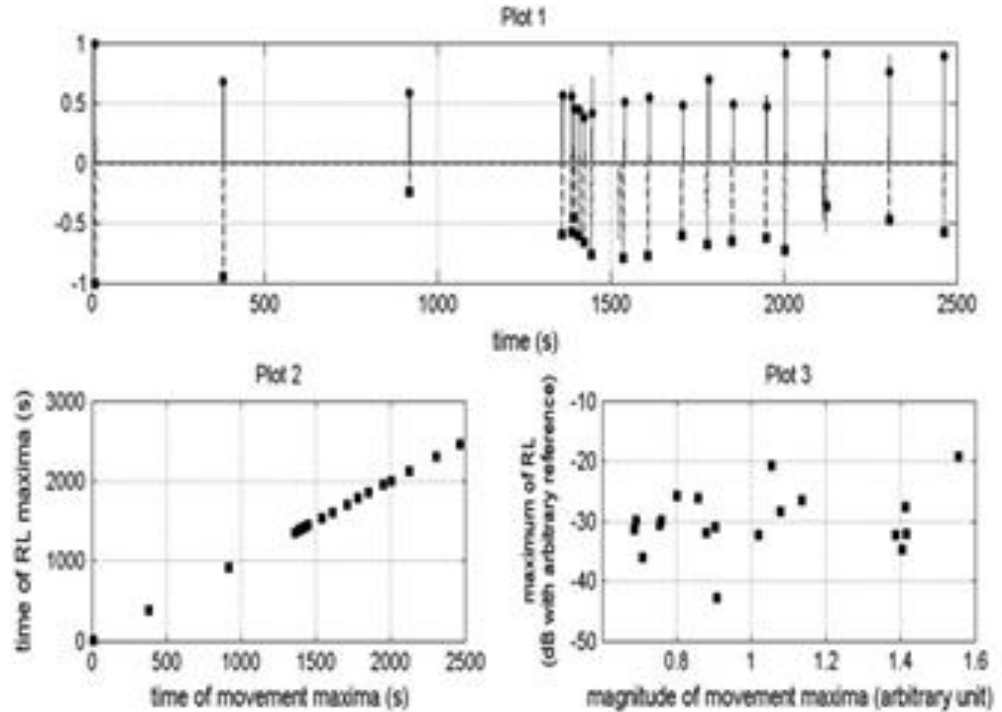

Fig 7:pilot insertion of MI channel network communication

and the grid inter-coil distance is presented. Furthermore, an approximation is given for the power loss between transmitter and receiver by clarifying the proportionality of the Performance dependence on coil parameters. Increasing the number of layers from 1 to 12 brings an improvement of $\approx 40 \mathrm{dBm}$ transmit power required to form a fully connected network. The distance dependent bandwidths, i.e., min-max bandwidths (Bmin, Bmax) between coils, are observed in fig 6 . 

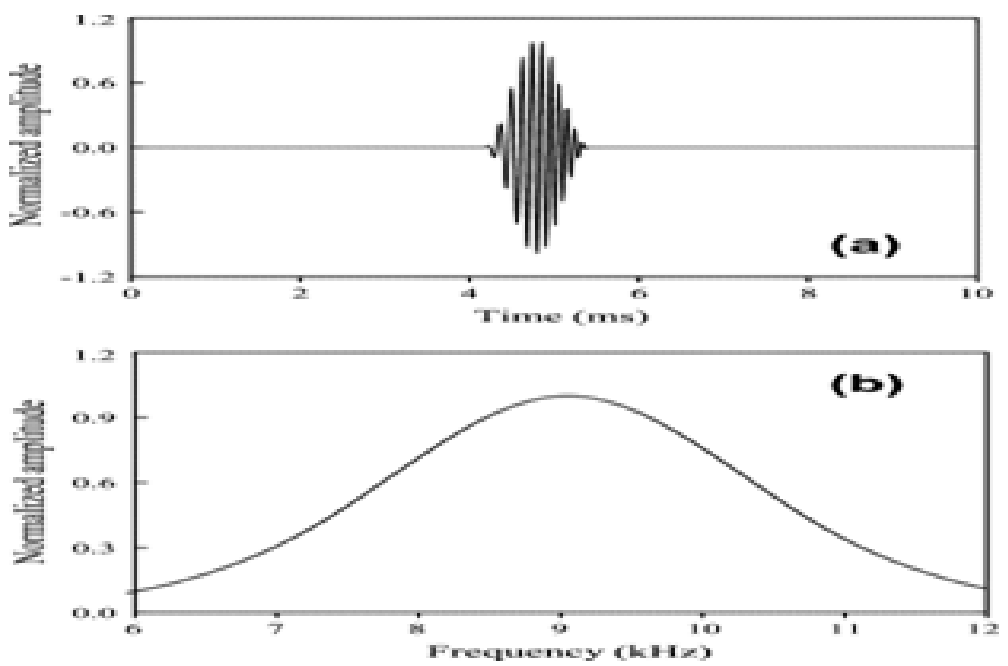

Fig 8: maximum and minimum of increased normalised amplitude Vs time in MI channel

The below table(9) shows that comparing with other underwater wireless communication methods, i.e., acoustic, optical and RF; it permits long-range communication as a promising alternative to acoustic communication. The comparison of required power, transmission range, propagation speed and bit per joule $(\mathrm{B} / \mathrm{E})$ is shown in Table for state of the art underwater wireless communication technologies where the values for MI are approximated. Although coil size can be adjusted to the range of interest for MI waves and the relaying topology changes the performance Significantly, for short range, although tens of Kbit/s data rate is very small compared with optical communication, it has a significantly large B/E ratio as shown. The dependence of performance on coil parameters and the grid inter-coil distance is analyzed.

TABLE I

comparison of underwater wireless communication channel

\begin{tabular}{|c|c|c|c|c|}
\hline $\operatorname{Sec} 1$ & \multicolumn{4}{|c|}{ Simp? } \\
\hline 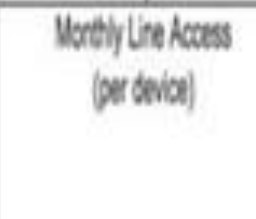 & $\begin{array}{l}\text { Stad } \\
\text { Vnts }\end{array}$ & $\begin{array}{l}\text { Send } \\
\text { Uessegos }\end{array}$ & Serd & 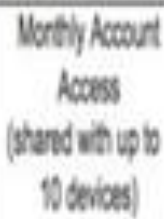 \\
\hline \multirow{6}{*}{ 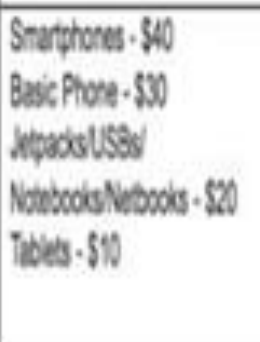 } & Uhinity & Uninitid & 108 & 550 \\
\hline & Whinixd & Winine & 268 & 80 \\
\hline & Uhinix & Whinat & 468 & 50 \\
\hline & Uhinied & Whink & $6 C 6$ & 80 \\
\hline & Uhinive & Whinitid & 808 & 50 \\
\hline & Whiniw & Uhinited & $10 G 9$ & 5100 \\
\hline
\end{tabular}

\section{CONCLUSION}

Underwater MI wireless communication networks using Induction coils are presented as promising candidates for underwater communication. SNR performance of 3D underwater networking topologies is theoretically modeled. 3D grid topologies are introduced for shallow and deep water, small and large area coverage networks. Furthermore, SNR, BER, connectivity and bandwidth performances of the proposed networks are numerically analyzed showing that between a few and tens of $\mathrm{KHz}$ wireless communication is possible with significantly high SNR for networking areas reaching to a few $\mathrm{km} 2$ in deep sea by forming a fully connected and power efficient multi-coil network. It improves the accuracy and to improve the bandwidth of underwater communication in wireless channels. 


\section{REFERENCES}

[1]. I. F. Akyildiz, D. Pompili, and T. Melodia, "Underwater acoustic sensor networks: Research challenges," Ad Hoc Networks, vol. 3, no. 3, pp. 257-279, 2005.

[2]. J.-H. Cui, J. Kong, M. Gerla, and S. Zhou, "Challenges: building scalable mobile underwater wireless sensor networks for aquatic applications," IEEE Network, vol. 20, no. 3, pp. 12-18, 2006.

[3]. X. Che, I. Wells, P. Kear, G. Dickers, X. Gong, and M. Rhodes "A static multi-hop underwater wireless sensor network using RF electromagnetic communications," in Proc. 2009 IEEE Int. Conf. On Distributed Computing Systems Workshops, pp. 460-463.

[4]. M. Stojanovic, "Underwater wireless communications: Current achievements and research challenges," IEEE Oceanic Engineering Society Newsletter, vol. 41, no. 2, 2006.

[5]. L. Butler, "Underwater radio communication," Amateur Radio, Apr. 1987.

[6]. I. Vasilescu, K. Kotay, D. Rus, M. Dunbabin, and P. Corke, "Data collection, storage, and retrieval with an underwater sensor network," in Proc. 2005 ACM Int. Conf. on Embedded Networked Sensor Systems, pp. 154-165.

[7]. Z. Sun and I. Akyildiz, "Magnetic induction communications for wireless underground sensor networks," IEEE Trans. Antennas Propag., vol. 58, no. 7, pp. 2426-2435, 2010.

[8]. Z. Sun and I. Akyildiz, "Underground wireless communication using magnetic induction," in Proc. 2009 IEEE International Conference on Communications, pp. 1-5.

[9]. R. Bansal, "Near-field magnetic communication," IEEE Antennas Propag. Mag., vol. 46, no. 2, pp. 114 115, 2004.

[10]. S. Meybodi, J. Nielsen, J. Bendtsen, and M. Dohler, "Magneto-inductive underground communications in a district heating system," in Proc. 2011 IEEE Int. Conf. on Communications, pp. 1-5.

[11]. Z. Sun and I. Akyildiz, "Deployment algorithms for wireless underground sensor networks using magnetic induction," in Proc. 2010 IEEE Global Telecommunications Conf., pp. 1-5.

[12]. Z. Sun, P. Wang, M. Vuran, M. Al-Rodhaan, A. Al-Dhelaan, and I. Akyildiz, "MISE-PIPE: magnetic induction-based wireless sensor networks for underground pipeline monitoring," Ad Hoc Networks, vol. 9, no. 3, pp. 218-227, 2011.

[13]. S. Kim, Y. Won, Y. Lim, K. Seo, and S. Lim, "Design of physical layer for magnetic field area network," in Proc. 2009 IEEE Int. Conf. on Ubiquitous Information Technologies and Applications, pp. 1-4.

[14]. Y. Won, S. Kang, K. Hwang, S. Kim, and S. Lim, "Research for wireless energy transmission in a magnetic field communication system," in Proc. 2010 IEEE Int. Symp. on Wireless Pervasive Computing, pp. 256-260.

[15]. I. Yoon and H. Ling, "Investigation of near-field wireless power transfer under multiple transmitters," IEEE Antennas Wireless Propag. Lett., vol. 10, no. 99, pp. 662-665, 2011.

[16]. J. Sojdehei, P. Wrathall, and D. Dinn, "Magneto-inductive (MI) communications," in Proc. MTS/IEEE Oceans Conf., vol. 1, pp. 513-519, 2001.

[17]. J. Agbinya and M. Masihpour, "Excitation methods for magneto inductive waveguide communication systems," in Proc. 2010 IEEE Broadband and Biomedical Communications, pp. 1-6. 